\title{
Models for the bead mobility technique: a droplet-based viscometer
}

Sellier, M. ${ }^{1}$ and Taylor, J.

Department of Mechanical Engineering, University of Canterbury, Private Bag 4800, Christchurch 8140, New Zealand

Betram, A.K.

Department of Chemistry, University of British Columbia, 2036 Main Mall, Vancouver BC, V6T 1Z1, Canada

Mandin, P.

Université de Bretagne Sud, IRDL - UMR CNRS 6027, Lorient, France

\section{Abstract}

Better understanding the properties of organic aerosols (OA) is attracting increasing attention because of the important role they play in climate change. The viscosity of OA has been shown to range from liquid to solid/semi-solid across the range of atmospheric relative humidity. A method known as the "bead-mobility technique" has been developed by Renbaum-Wolff et al. (RenbaumWolff, Grayson, and Bertram 2013) to quantify the viscosity of an atmospheric particle over a range of atmospherically relevant humidities. The method is based on the assumption that the strength of the flow recirculation inside a droplet placed in a shear flow is related to the droplet viscosity. This paper presents a simple analytical model which predicts the internal flow in the droplet and provides a correlation relating the strength of the flow in the droplet to its viscosity. The validity of this analytical model is assessed by comparing the analytical results with a corresponding two-phase flow simulation with a moving mesh which captures the motion of the interface. The ability of the analytical model to reproduce experimental data reported in (Renbaum-Wolff, Grayson, and Bertram 2013) is also quantified. The reasonable agreement between the analytical model and the experimental data confirms that the droplet velocity provides a useful proxy to estimate the droplet viscosity for small liquid samples for which standard viscometry techniques do not apply.

\section{Keywords}

Viscometry, shear flow, droplet, aerosols, two-phase flow, free surface flow

\section{Introduction}

According to the Intergovernmental Panel on Climate Change, aerosols have likely decreased the radiative forcing (cooling effect) of the earth-atmospheric system over the industrial period. However, this cooling effect is highly uncertain, leading to uncertainties in climate models (Solomon

\footnotetext{
${ }^{1}$ Corresponding author: mathieu.sellier@canterbury.ac.nz
} 
et al. 2007). Accurately representing organic aerosols (OA) in atmospheric models is one of the key requirements for understanding the climate effects of aerosols. The viscosity of OA has been shown to range from liquid to solid/semi-solid across the range of atmospheric relative humidity (RenbaumWolff et al. 2013). The viscosity of these atmospheric particles is currently a topic of interest since viscosity influences the environmental impacts of these particles. For example, the viscosity of OA affects the particles ability to accommodate water and act as cloud condensation nuclei or ice nuclei, influences the partitioning of water and gases, changes the atmospheric lifetime of the particles, and affects the rate of heterogeneous chemical reactions (Virtanen et al. 2010, Zaveri et al. 2018, Wang et al. 2012, Shiraiwa et al. 2011, Perraud et al. 2012). Determining the viscosity of OA particles is an especially challenging goal since the viscosity of OA can range from $10^{-3} \mathrm{~Pa}$.s (similar to water) to $10^{12}$ Pa.s (similar to a glass marble), as the relative humidity varies in the atmosphere. In addition, the amount of material that can be collected from the atmosphere or in environmental chambers which are used for simulating atmospheric conditions, is on the order of 1-5 mg (approximately 1-5 microlitres).

Classical macroscopic rheometry techniques require sample volumes of approximately $1 \mathrm{ml}$ and involve characteristic length scales of approximately $1 \mathrm{~mm}$ (Pipe and McKinley 2009). Such large consumptions of fluid is not appropriate for many biological and medical samples which are typically limited to a microliter (Han, Tang, and Zheng 2007). In order to decrease the amount of fluid required, many macro-scale devices have been adapted to incorporate micrometric scales. For example, (Srivastava and Burns 2006) developed a silicon-glass hybrid microfluidic device and (Müller and Pita 1983) scaled down the classical falling ball method to create a new microviscometer. Unfortunately, a common issue associated with the above two techniques is the expensive or complex setup of instrumentation that is required. (Han, Tang, and Zheng 2007) developed a PDMS (Polydimethylsiloxane) based microliter viscometer however has only been validated up to a viscosity $80 \mathrm{mPa}$.s. Nano scale rheometry can be studied through atomic force microscopy or surface force instruments which both require a small sample volume however instrument artefacts can overwhelm observations facilitating the need to introduce large correction factors in order to compensate for flow non-idealities. In addition, atomic force microscopy is limited to low viscosities ( $\leq 5 \times 10^{-2} \mathrm{~Pa}$.s) due to the strong dissipative effects which occur at higher viscosities (Pipe and McKinley 2009). A number of alternative viscometry techniques for organic aerosols have been proposed and analysed over the recent past. (Zhang et al. 2015) developed the "shape relaxation" method which applies for the viscosity range $10^{5}-10^{11}$ Pa.s. The "dimer relaxation" method proposed by (Rothfuss and Petters 2016) is applicable for $5 \times 10^{5}<\mu<$ $2 \times 10^{7}$ Pa.s. The "aerosol optical tweezers" pioneered by (Power and Reid 2014) covers the widest range of viscosity from $10^{-3}$ Pa.s to $10^{9}$ Pa.s. The validity of the "poke flow" method was assessed in (Grayson et al. 2015). The light scattering method of (Järvinen et al. 2016) applies to viscosities in the vicinity of $10^{7}$ Pa.s. Finally, the "Fluorescence lifetime imaging (FLIM)" of (Hosny et al. 2013) offers the prospect of estimating viscosities in the $10^{-3}-10^{3} \mathrm{~Pa}$.s range. Note that these techniques and others have recently been exhaustively reviewed in (Reid et al. 2018).

Although the above techniques are capable of studying the rheometry of very small samples, a common shortcoming is the limitation on the viscosity range. A technique able to incorporate a larger range, particularly higher viscosities is needed for applications such as the study of environmental chamber samples. The bead mobility technique is capable of determining viscosities in the range $\left(10^{-3}<\mu<10^{3} \mathrm{~Pa}\right.$.s $)$. This method allows real time control over relative humidity ( $\left.\mathrm{RH}\right)$ making it capable of simulating atmospheric conditions. This, combined with the small sample volume requirement and viscosity range, makes this technique highly applicable to the study of OA 
particles. Through this method, viscosities of several types of organic aerosol produced in environmental chambers have been studied. For example, the viscosity of organic aerosol produced by the ozonolysis of $\alpha$-pinene has been quantified for relative humidities between $70 \%$ and $90 \%$, and the viscosities of the particles were found to range from $791 \mathrm{~Pa} . \mathrm{s}$ (comparable to peanut butter) at 70\% RH to 6.25 Pa.s (comparable to honey) at 90\% RH (Renbaum-Wolff et al. 2013, Song et al. 2015, Song et al. 2016).

As a brief summary of the technique, particle material is reduced to a fine mist (atomised) and sprayed onto a slide or alternatively the particle material is deposited directly on a slide using an impactor. Both processes result in super-micron droplets, typically $20 \mu \mathrm{m}$ to $50 \mu \mathrm{m}$ in diameter, of the sample fluid collected on a slide. In order to visualize the internal circulation of the fluid inside the droplets, a dilute suspension of hydrophilic melamine beads is sprayed over the slide and allowed to settle into the sample droplets. The slide is then placed inside a flow cell with a gas flow velocity of approximately $1 \mathrm{~m} / \mathrm{s}$. The gas is constituted of a mixture of nitrogen and water with the relative humidity of the flow being controlled in real time. The movement of the gas over the droplets generates a shear stress at the surface of the droplets, causing the fluid within the droplet to circulate. The movement of the beads within the droplets is tracked using an optical microscope and used to calculate an average bead velocity. The bead velocity is then used to calculate the droplet viscosity based on a calibration curve constructed using standards with known viscosities. At the core of this investigation is the shear-driven flow inside a droplet. This problem has attracted the attention of several researchers over the years either numerically (Pozrikidis 1997, Spelt 2006), analytically (Dussan 1987, Sugiyama and Sbragaglia 2008, Woodhouse and Goldstein 2012), or experimentally (Fan, Wilson, and Kapur 2011, Mahé et al. 1988).

As it is, the bead mobility technique requires a calibration curve. The purpose of this paper is to present a validated, analytical model which provides a simple approximate correlation between the velocity field in the droplet and the viscosity. This could potentially circumvent the need for the time-consuming generation of a calibration curve and will reveal explicitly the effects of various parameters such as droplet size, external flow velocity, contact angle, etc ...

The paper is organized as follows. Section 2 describes both the analytical and numerical models. The validity of the analytical model is assessed and discussed in Section 3 and concluding remarks are presented in section 4.

\section{Analytical and numerical models}

\section{a. Assumptions and key parameters}

In this simplified model, the flow is assumed to be planar and the fluids are assumed to be Newtonian and incompressible. The droplet rests on the lower surface of a flow cell through which a constant flow rate is maintained. Figure 1 shows a sketch of the problem considered and corresponding notations. The flow cell height is $H$ and its length is $L$. The gas in the flow cell has kinematic viscosity $v_{g}$ and density $\rho_{g}$. The two-dimensional droplet is assumed to be a circular segment of area $A$, radius of curvature $R_{d}$, and contact angle $\theta$. The corresponding radius of the droplet footprint on the substrate is $R_{d}$, as illustrated in Fig. 1 . The liquid in the droplet has density $\rho$, dynamic viscosity $\mu$, and interfacial tension $\sigma$. The free surface of the droplet is described by the thickness $h(x, t)$. The location of the free surface is in principle a function of time but we will consider steady-state solutions in the following. Because the typical size of the droplet is very small, it is safe to neglect the effect of gravity (low Bond number regime) and the combination of the small 
droplet size and high viscosity of the liquid forming the droplets implies a low Reynolds number inner flow, i.e. creeping flow conditions prevail within the droplets. The creeping flow regime motivates the following analysis based on the lubrication approximation.

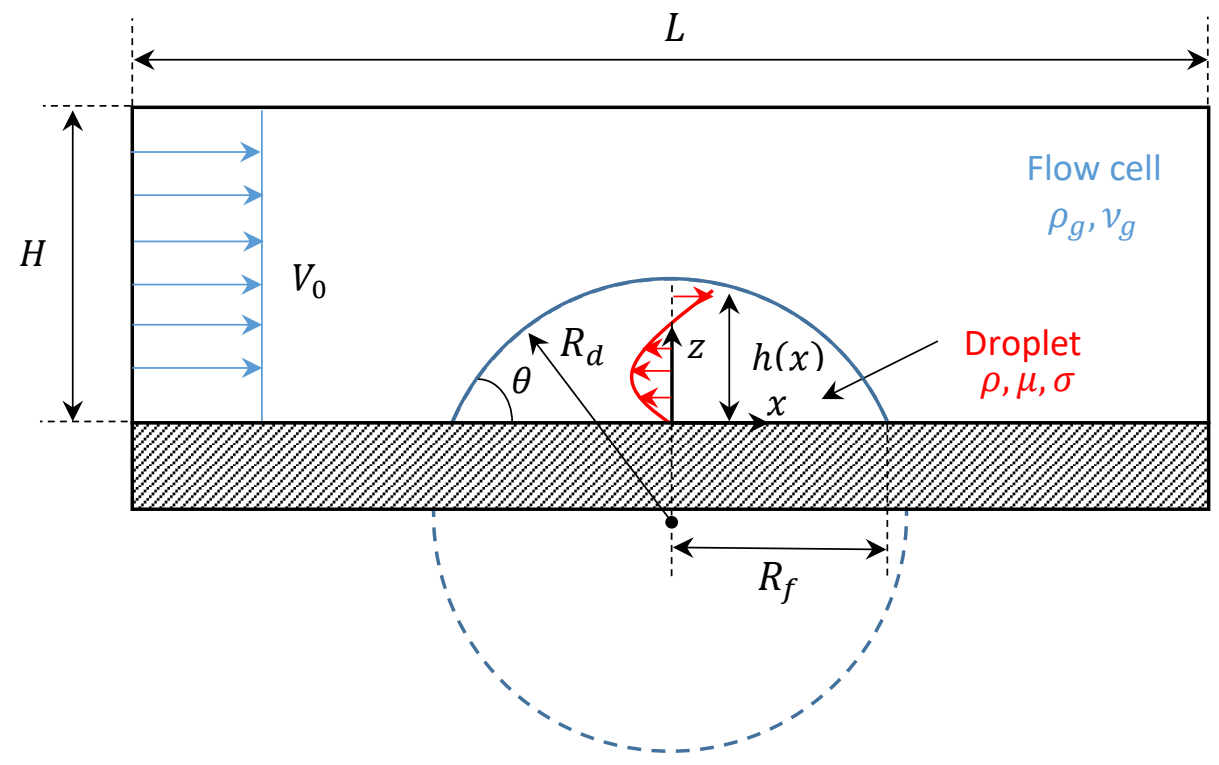

Figure 1: Sketch of the problem considered and notations

\section{b. Flow inside the droplet}

The momentum conservation equation in the $\mathrm{x}$-direction is simplified based the assumption that inertial effects are negligible and the flow is effectively unidirectional, typical assumptions of the lubrication approximation. The resulting momentum equation in the $x$-direction balances viscous stresses and the pressure gradient within the droplet. Accordingly

$$
\frac{\partial}{\partial z}\left(\mu \frac{\partial u}{\partial z}\right)=\frac{\partial p}{\partial x}
$$

where $u(x, z, t)$ is the velocity in the $x$-direction and $p(x, z, t)$ the pressure distribution. This differential equation is subject to no-slip on the solid surface $u(x, z=0, t)=0$ and the droplet surface is subject to the shear stress $\tau_{s}$ generated by the flow over the droplet such that $\left.\mu \frac{\partial u}{\partial z}\right|_{z=h}=$ $\tau_{s}$. The velocity profile which satisfies eq. (1) and boundary conditions is parabolic and given by

$$
u(x, z, t)=\frac{1}{\mu} \frac{\partial p}{\partial x}\left(\frac{z^{2}}{2}-z h\right)+\frac{\tau_{s} z}{\mu}
$$

At this stage, the equation contains two unknowns: the pressure gradient $\frac{\partial p}{\partial x}$ and the shear stress $\tau_{s}$ induced by the gas at the surface of the droplet. In order to find an expression for the pressure gradient, we can apply the conservation of mass. If we assume the system has reached a steady state and therefore the droplet shape is invariant in time, the net mass flow rate through any crosssection must vanish. Accordingly, the condition

$$
\int_{0}^{h} u(x, z, t) d z=0
$$

leads to $\frac{\partial p}{\partial x}=\frac{3 \tau_{s}}{2 h}$. Therefore, the velocity profile inside the droplet simplifies to 


$$
u(x, z, t)=\frac{1}{\mu} \frac{3 \tau_{s}}{2 h}\left(\frac{z^{2}}{2}-z h\right)+\frac{\tau_{s} z}{\mu}
$$

We can therefore evaluate the free surface velocity

$$
u(x, z=h, t)=\frac{\tau_{s} h}{4 \mu}
$$

The free surface velocity is therefore proportional to the applied shear stress and the droplet thickness $h$ but inversely proportional to the droplet viscosity.

\section{c. Shear stress at the droplet surface}

Eq. (4) is still defined in terms of the unknown shear stress on the droplet. Should the substrate be flat, i.e. free of droplets, the substrate would experience a wall shear stress which can be calculated using the following friction factor correlation (Fan, Wilson, and Kapur 2011)

$$
f=\frac{2 \tau_{w}}{V_{0}^{2} \rho_{g}}= \begin{cases}16 / R e & R e<500 \\ 0.0792 R e^{1 / 4} & R e \geq 500\end{cases}
$$

where $R e=\frac{V_{0} D_{H}}{v_{g}}$ is the flow cell Reynolds number with $D_{H}$ the hydraulic diameter of the flow cell.

The presence of the droplet will affect the local flow and therefore the local shear stress distribution. In order to quantify this effect, we use the results of (Pozrikidis 1997, Sugiyama and Sbragaglia 2008) to evaluate an effective, average shear stress on the droplet. From (Pozrikidis 1997), the total drag force exerted by the flow on spherical cap of radius of curvature $a$ is

$$
F_{d}=C \mu k \pi a^{2}=C \tau_{w} \pi a^{2}
$$

where $C$ is a constant which depends on the contact angle, $k$ the velocity gradient at the wall, and therefore $\mu k=\tau_{w}$. The constant $C$ is equal to 4.3 for a hemisphere but could be as high as 10.2 for a sphere (see Table 1 of (Pozrikidis 1997)). The effective, average shear stress on the droplet $\tau_{s}$ is defined here as the average shear stress acting on the surface area of the droplet footprint which produces in the same amount of drag. Accordingly,

$$
\pi R_{f}^{2} \tau_{s}=C \tau_{w} \pi a^{2}
$$

where $R_{f}$ is the radius of the droplet footprint. Equivalently,

$$
\tau_{s}=C\left(\frac{a}{R_{f}}\right)^{2} \tau_{w}
$$

\section{d. Numerical model}

The problem at hand is clearly a two-phase flow problem involving two immiscible fluids. The droplet has a deformable free surface and a mobile contact line. In order to model such a problem, we use the "Laminar two-phase flow moving mesh" interface in COMSOL Multiphysics. This interface combines a Finite Element Navier-Stokes solver with a moving mesh module which allows the mesh to deform with the interface. An additional set of partial differential equations is solved for the mesh displacement and redistribution of internal nodes. This module is well suited to model capillary flows 
with a contact line. A Navier-slip boundary condition is applied at the contact line to circumvent the well-known "contact line paradox" (Huh and Scriven 1971).

The computational domain considered here is two-dimensional. It can trivially be extended to threedimension but it is not necessary for the forthcoming discussion and the additional computational cost is significant. A uniform velocity profile is imposed at the inlet and the pressure is set to zero at the outlet. Standard dynamic and kinematic boundary conditions are applied at the droplet free surface where the interfacial tension $\sigma$ is specified. A no-slip boundary condition is applied on the upper and lower wall of the flow cell and a Navier-Slip boundary condition is applied on the portion of the wall which is wetted by the droplet. The initial droplet domain is set to be a circular segment with prescribed volume $A$ and contact angle $\theta$. Boundary conditions are also required for the moving mesh module. Displacement are set to zero on all boundaries which do not experience any motion (inlet, outlet, upper and lower walls) whereas the liquid/gas interface is set as a free surface which is free to deform.

An unstructured mesh is generated which contains $~ 75,000$ P1-P1 triangular elements for the fluid flow problem ("Extra-Fine" mesh setting). The Winslow mesh smoothing technique is employed to deform the mesh when the interface deforms. The problem is solved as a transient simulation until a steady state is reached. The transient discretization uses the Generalized-alpha method with variable time-stepping.

The validity of the numerical model was assessed by comparing the numerical results to the previously described simplified analytical model as described in the next section.

\section{Results and discussion}

\section{a. Baseline study}

As a baseline study representative of the forthcoming comparison with experimental data, we consider a droplet of oil (represented by a circular segment in these planar simulations) with radius $R_{d}=25 \mu \mathrm{m}$, viscosity $\mu=0.79$ Pa.s, density $\rho=888 \mathrm{~kg} / \mathrm{m}^{3}$, contact angle $\theta=90^{\circ}$, and interfacial tension $\sigma=0.07 \mathrm{~Pa}$. $\mathrm{m}$ immersed in air with kinematic viscosity $v_{g}=1.51 \times 10^{-5} \mathrm{~m}^{2} / \mathrm{s}$ and density $\rho_{g}=$ $1.2 \mathrm{~kg} / \mathrm{m}^{3}$. The flow cell height $H$ is 4 times the maximum droplet height, and its extent $L$ is 20 times the droplet radius. The inlet velocity $V_{0}$ is $0.01 \mathrm{~m} / \mathrm{s}$. The corresponding outer flow Reynolds number is $R e=\frac{V_{0} D_{H}}{v_{g}}=\frac{V_{0} H}{v_{g}}=6.6225 \times 10^{-2}$. The inner flow Reynolds number based on the droplet diameter, the maximum velocity and minimum viscosity is approximately $10^{-8}$ justifying the earlier assumption that inertia can safely be neglected.
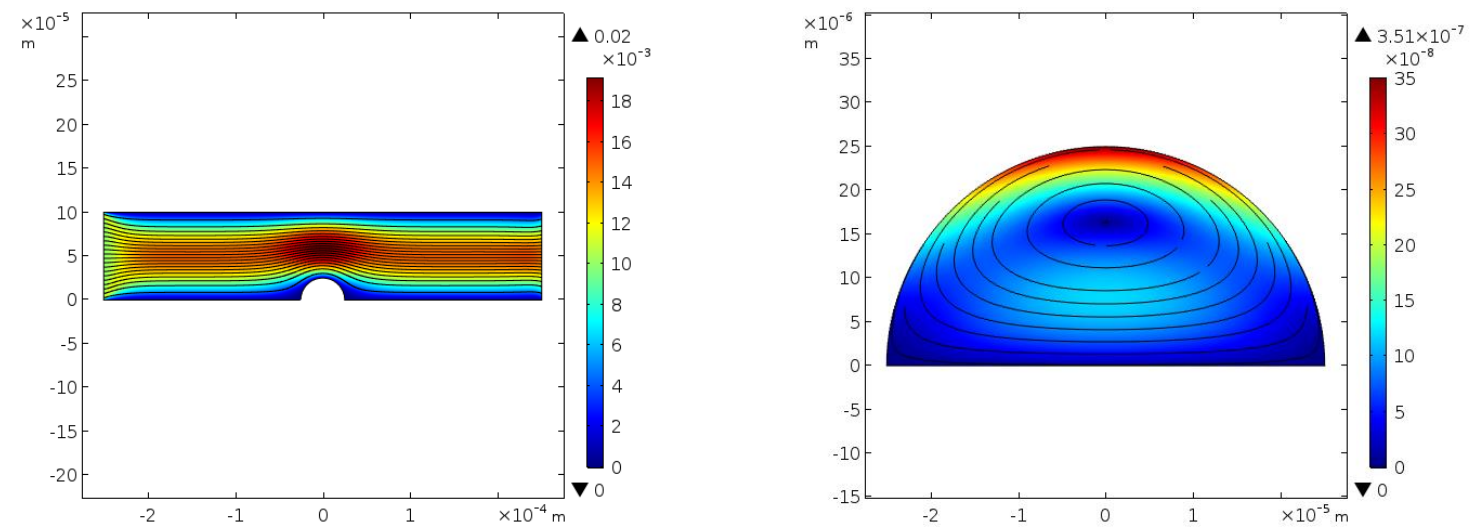
Figure 2: Flow streamlines and contours of velocity magnitude for the outer flow (left-hand-side) and inner flow (right-handside) at $t=30 \mathrm{~s}$.

Figure 2 illustrates the flow field in the flow cell and the droplet at $t=30 \mathrm{~s}$ when the flow has reached a steady-state. Because the outer flow Reynolds number is small, the flow is seen to remain attached to the droplet. Streamlines converge on the fore side of the droplet indicating an accelerating flow, classical features of the cross-flow over a cylinder. The streamlines inside the droplet confirm the existence of a recirculating flow. The stagnation point at the centre of this recirculation is located in the droplet fore/aft symmetry plane. (LeClair et al. 1972) have shown that this stagnation point is slightly off-centred for larger entrainment velocities. Note that the magnitude of the velocity in the droplet is four orders of magnitude smaller than that in the flow cell. Of course, this is expected given the corresponding orders of magnitude difference in dynamic viscosity between oil and air. Eq. (6) predicts a wall shear stress $\tau_{w}$ of $1.45 \times 10^{-2} \mathrm{~Pa}$. An average of the viscous shear stress on the upper wall in the numerical simulation gives $1.35 \times 10^{-2} \mathrm{~Pa}$, a reasonably close value given that the flow is not full developed at the inlet and disturbed by the presence of the droplet. On the other hand, Figure 3 showing the wall shear stress upstream of the droplet clearly indicates that eq. (6) yields a fairly poor prediction of the wall shear stress experienced by the droplet.

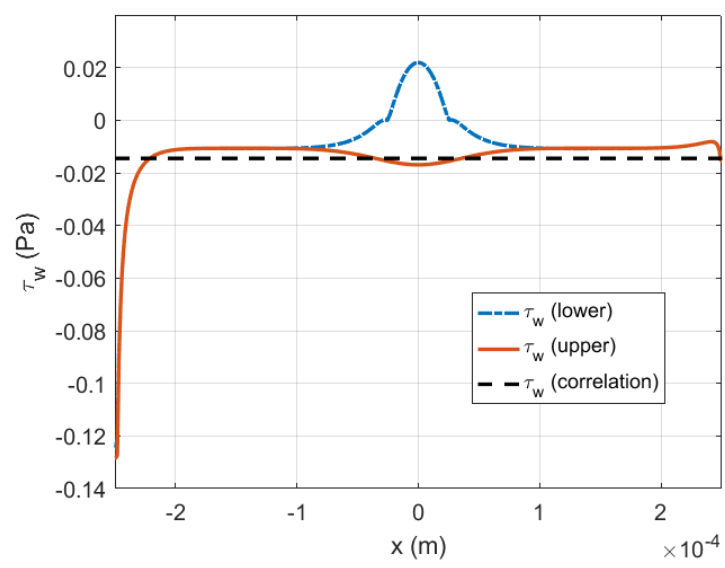

Figure 3: wall shear stress distribution on the upper and lower wall of the flow cell. The black line represents the wall shear stress predicted by correlation (6)

For these flow conditions, simulations show that the droplet did not experience any significant deformation and therefore $a=R_{f}=R_{d}$. Using eq. (9), we find that the effective, average shear stress $\tau_{s}$ experienced by the droplet is $6.32 \times 10^{-2} \mathrm{~Pa}$ ( 4.3 times the shear stress that the wall would experience in the absence of the droplet). Using eq. (4), we can then estimate the velocity profile along the droplet centreline $(x=0)$. This estimated velocity profile is compared to the one computed numerically in Figure 4 (left-hand-side). Both curves agree qualitatively: they follow the same trend but the analytical solution over-predicts the numerical solution by $\sim 42 \%$ at the droplet apex. The velocity distribution on the droplet free surface is calculated using eq. (5) and then compared to the numerically predicted one on Figure 4 (right-hand-side). One notes that the velocity distribution is symmetrical as one would expect for this very low Reynolds number. Again, the distributions agree qualitatively but feature a difference in magnitude which peaks at the droplet apex. This difference is not unexpected since the large contact angle $\left(\theta=\frac{\pi}{2}\right)$ falls outside the expected validity range of the lubrication approximation which assumes that the free surface has a small slope. 

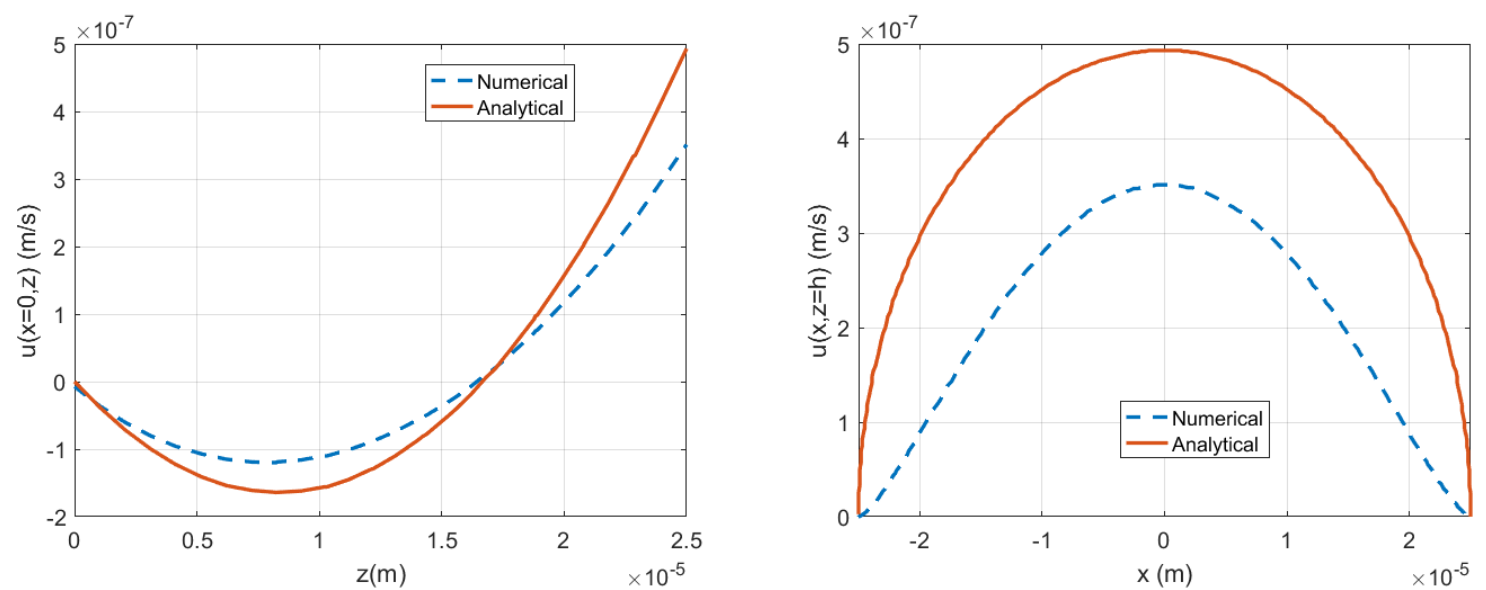

Figure 4: Velocity distribution on the centreline $x=0$ (left-hand-side) and on the droplet free surface from the fore to the aft stagnation points (right-hand-side)

Nonetheless, using the effective wall shear stress (eq. (9)) instead of the wall shear stress of the droplet-free surface (eq. (6)) considerably improves the agreement between the analytical and numerical velocities and suggests that the analytical model is able give a sound approximation of the flow field inside the droplet. It is therefore likely to provide a reasonable proxy to estimate the droplet viscosity for given bead velocity observations.

\section{b. Parametric study}

In order to test the validity of the simple proposed analytical model, we performed a parametric study. Using the previous case study as the baseline, we varied the velocity of the incoming air, the viscosity of the liquid droplet, and the contact angle but kept all other parameters constant including the area of the circular segment $A\left(\frac{\pi R_{f}^{2}}{2}=\frac{\pi R_{d}^{2}}{2}=9.81 \times 10^{-10} \mathrm{~m}^{2}\right.$ for the baseline case). The area of the circular segment was kept the same but the contact angle varied using simple trigonometric formulae ${ }^{2}$. This resulted in the Reynolds number $R e$ ranging from $3.7 \times 10^{-2}$ to 6.6 , the contact angle $\theta$ taking the values $\left[\frac{\pi}{6}, \frac{\pi}{3}, \frac{\pi}{2}\right]$, and the viscosity ratio $\lambda=\frac{\mu}{\mu_{g}}$ spanning 2 orders of magnitude from $4.36 \times 10^{3}$ to $4.36 \times 10^{5}$. Results are summarized in Table 2 in the Appendix and illustrated in Fig. 5 which shows the apex velocity calculated analytically $\left(u_{\text {apex }}^{\text {ana }}\right)$ plotted against that computed in the numerical model $\left(u_{\text {apex }}^{\text {num }}\right)$. The excellent agreement between the cloud of data and the identity line $y=x$ confirms that the simple analytical model provides a reliable estimate of the droplet apex velocity over a wide range of parameters. The ratio $\frac{u_{a p e x}^{a n a}}{u_{a p e x}^{n u m}}$ is found to belong to the range $[0.74,1.42]$. The lower end of this range occurs when $\theta=\frac{\pi}{6}$ while the upper end occurs when $\theta=\frac{\pi}{2}$. The best results in terms of agreement between the numerical and analytical model are for $\theta=\frac{\pi}{3}$. Within the parameter range considered, the numerical results confirm that as predicted by the simple analysis:

1. for a given outer flow Reynolds number and contact angle the apex velocity is inversely proportional to the droplet viscosity

2. for a given viscosity and contact angle, the apex velocity is proportional to the outer flow velocity $V_{0}$

\footnotetext{
${ }^{2}$ http://mathworld.wolfram.com/CircularSegment.html
} 


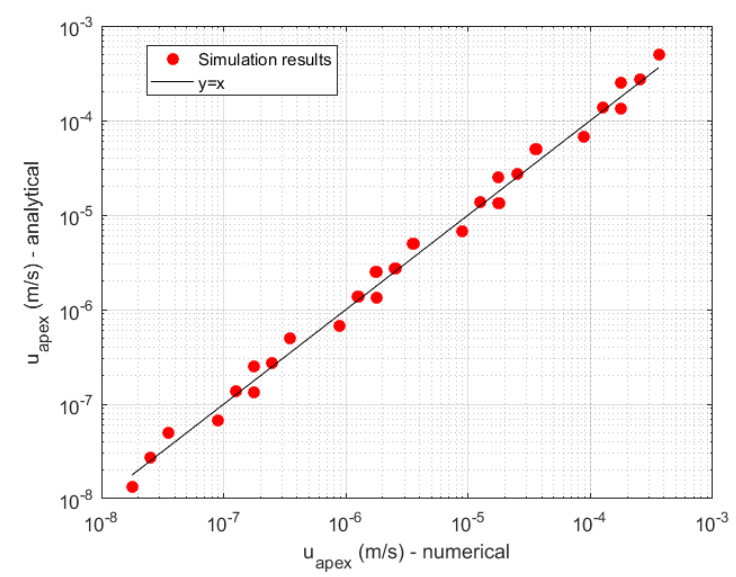

Figure 5: Log-log plot of the apex velocity predicted analytically against the apex velocity computed numerically

\section{c. Comparison with experimental data}

In the experiments presented in $[1,4]$, nitrogen with a small amount of water to control humidity flows into the in the flow cell. Hereafter, the outer gas properties are assumed to be that of nitrogen at $22.5^{\circ} \mathrm{C}$ under 1 bar pressure, i.e. density $\rho_{g}=1.1404 \mathrm{~kg} / \mathrm{m}^{3}$ and kinematic viscosity $v_{g}=$ $15.542 \times 10^{-6} \mathrm{~m}^{2} / \mathrm{s}$. The density of the droplet $\rho_{d}=1000 \mathrm{~kg} / \mathrm{m}^{3}$ is defined as the value adopted by Pathak et al [29]. Two ranges of viscosities have been tested. Firstly, the viscosity range studied during the bead mobility tests of $\alpha$-pinene particles $\left(16.3<\mu_{d}<791\right.$ Pa.s), and secondly, the range of viscosities used to define the calibration curve $\left(10^{-3}<\mu_{d}<10^{3}\right.$ Pa.s $)$ as presented by RenbaumWolff et al $[1,4]$. The calibration curve was generated by measuring the bead velocity for droplets of known viscosity. Renbaum-Wolff et al (Renbaum-Wolff, Grayson, and Bertram 2013) reported droplet diameters to range between $30 \mu \mathrm{m}$ and $50 \mu \mathrm{m}$. The analytical solution will therefore be formulated for an average droplet diameter of $40 \mu \mathrm{m}$ (i.e. $R_{d}=20 \mu \mathrm{m}$ ) and a contact angle $\theta=\frac{\pi}{2}$. Unfortunately, the flow cell used in the experiments has a complex shape as shown in Figure 6 (lefthand-side) which makes the evaluation of the wall shear stress more challenging.
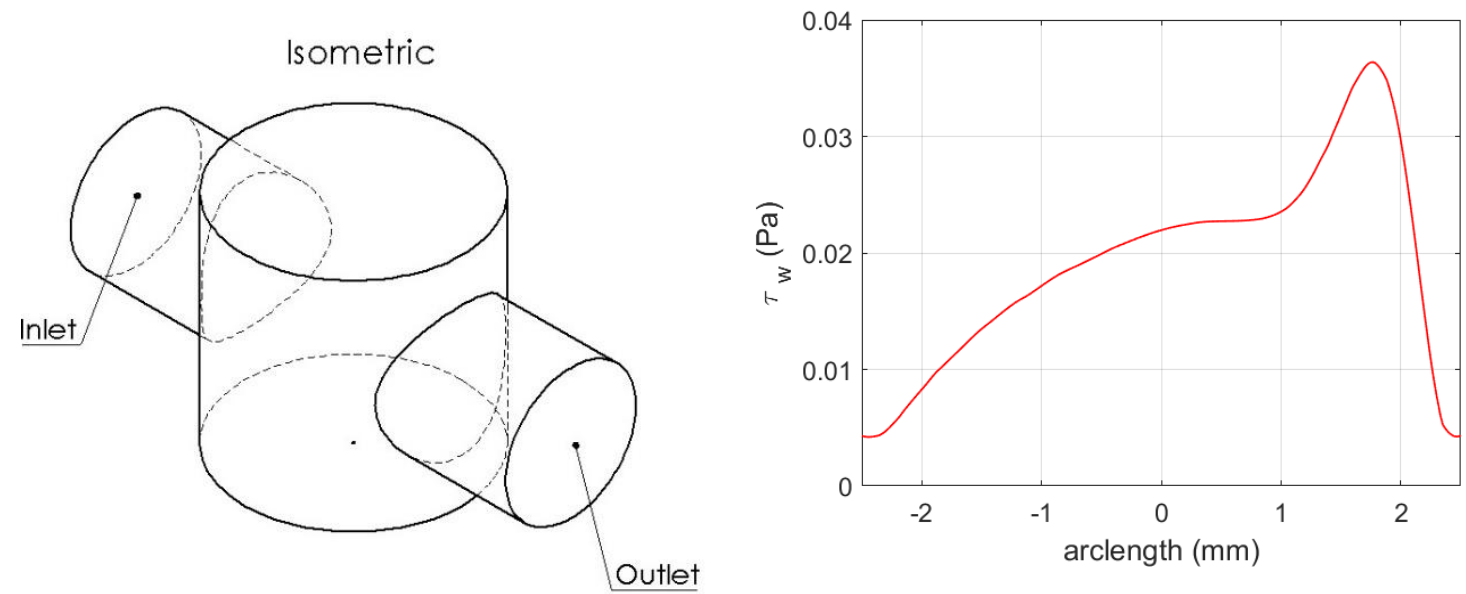

Figure 6: Flow cell used in (Renbaum-Wolff, Grayson, and Bertram 2013) (left-hand-side) and shear stress distribution on the lower wall on the centreline (right-hand-side)

The volumetric flow rate through the flow cell was $1.2 \mathrm{l} / \mathrm{min}$. The diameter of the inlet port was $3 \mathrm{~mm}$. The corresponding average velocity and Reynolds number are therefore $2.829 \mathrm{~m} / \mathrm{s}$ and 546 , 
respectively. Given the $5 / 3$ expansion ratio of the flow cell, we estimate that the velocity $V_{0}$ felt by the droplet will be $1.02 \mathrm{~m} / \mathrm{s}$ (velocity decreased by $(5 / 3)^{2}$ ). Using eq. (6), the corresponding wall shear stress will be approximately $9.7 \times 10^{-3} \mathrm{~Pa}$ and therefore, the effective, average shear stress experienced by the droplet would be $4.17 \times 10^{-2} \mathrm{~Pa}$. We can then apply eqs. (4) and (5) to calculate the expected velocity in the droplets. Note that experiments reported in (Renbaum-Wolff, Grayson, and Bertram 2013) used an average velocity which will be lower than the apex velocity but in the absence of a reliable correlation between the two, we will use the apex velocity to calculate the expected bead velocity for a given viscosity. The calibration curve fitted by Renbaum-Wolff et al. (Renbaum-Wolff, Grayson, and Bertram 2013) used a power law of the form $u_{\text {bead }}=a \mu^{b}$, where $(a, b)=\left(2.73 \times 10^{-7},-0.955\right)$ are fitting constants using the least squares method. The corresponding $R^{2}$ value is 0.988 . The correlation based on eq. (5) gives $(a, b)=\left(2.08 \times 10^{-7},-1\right)$ which are reasonably close values. The comparison between the analytical predictions and the experimental results is shown in Table 1. The table shows the measured and predicted velocity for a range of viscosities.

\begin{tabular}{|c||c||c|c||c|c||c|c|}
\multicolumn{1}{c||}{} & $\begin{array}{c}\text { Analytical } \\
\text { solution }\end{array}$ & \multicolumn{2}{c||}{$\begin{array}{c}\text { Calibration curve } \\
\text { From (Renbaum- } \\
\text { Wolff, Grayson, and } \\
\text { Bertram 2013) }\end{array}$} & $\begin{array}{c}\text { HEC } \\
\text { From (Renbaum- } \\
\text { Wolff, Grayson, and } \\
\text { Bertram 2013) }\end{array}$ & $\begin{array}{c}\text { PNNL } \\
\text { From (Renbaum- } \\
\text { Wolff, Grayson, and } \\
\text { Bertram 2013) }\end{array}$ \\
\hline $\begin{array}{c}\text { Viscosity } \\
\text { (Pa.s) }\end{array}$ & $\begin{array}{c}\text { Bead } \\
\text { velocity } \\
(\mathrm{m} / \mathrm{s})\end{array}$ & $\begin{array}{c}\text { Bead } \\
\text { velocity } \\
(\mathrm{m} / \mathrm{s})\end{array}$ & $\begin{array}{c}\text { Error } \\
(\%)\end{array}$ & $\begin{array}{c}\text { Bead } \\
\text { velocity } \\
(\mathrm{m} / \mathrm{s})\end{array}$ & $\begin{array}{c}\text { Error } \\
(\%)\end{array}$ & $\begin{array}{c}\text { Bead } \\
\text { velocity } \\
(\mathrm{m} / \mathrm{s})\end{array}$ & $\begin{array}{c}\text { Error } \\
(\%)\end{array}$ \\
\hline 0.001 & $2.08 \times 10^{-4}$ & $2.0 \times 10^{-4}$ & 4.17 & & & & \\
\hline 6.25 & $3.33 \times 10^{-8}$ & $5.0 \times 10^{-8}$ & 33.3 & $4.75 \times 10^{-8}$ & 29.8 & & \\
\hline 16.3 & $1.28 \times 10^{-8}$ & $2.0 \times 10^{-8}$ & 36.1 & $1.90 \times 10^{-8}$ & 32.7 & $1.90 \times 10^{-8}$ & 32.7 \\
\hline 488 & $4.27 \times 10^{-10}$ & $7.9 \times 10^{-10}$ & 46 & $7.40 \times 10^{-10}$ & 42.3 & $7.41 \times 10^{-10}$ & 42.4 \\
\hline 791 & $2.63 \times 10^{-10}$ & $5.0 \times 10^{-10}$ & 47.3 & $4.67 \times 10^{-10}$ & 43.6 & & \\
\hline 1000 & $2.08 \times 10^{-10}$ & $4.0 \times 10^{-10}$ & 47.9 & & & & \\
\hline
\end{tabular}

Table 1: Comparison of average bead speeds between analytical solution and experimental results. HEC and PNNL represent data obtained from two separate experiments at HEC and PNNL labs measuring $\alpha$-pinene particles. The calibration curve was constructed using fluids of known viscosity.

For the lowest viscosity, the analytical model predicts the bead velocity very well with an error defined as $100\left|\frac{u_{\text {bead }}-u_{\text {analytical }}}{u_{\text {bead }}}\right|$ of only $4.17 \%$ when compared to the experimental correlation. The error increases with viscosity to reach $47.9 \%$ at the highest droplet viscosity of 1000 Pa.s. The analytical solution tends to under-predict the bead velocity but the analytical expression still provides a very useful proxy to determine the droplet viscosity given its simplicity and the paucity of other reliable methods to estimate the viscosity of such small, highly viscous liquid samples.

Given the analytical correlation eq. (5), an uncertainty analysis is now performed to explain the difference between the model predictions and the experimental data. Eq. (9) implies that $\frac{\Delta \tau_{s}}{\tau_{S}} \sim \frac{\Delta C}{C}+$ $\frac{\Delta \tau_{w}}{\tau_{w}}$ while we can deduce from eq. (5) that $\frac{\Delta u}{u} \sim \frac{\Delta \tau_{s}}{\tau_{s}}+\frac{\Delta h}{h}+\frac{\Delta \mu}{\mu}$. From the above expressions, we infer that the total uncertainty on the estimated viscosity will be $\frac{\Delta \mu}{\mu} \sim \frac{\Delta \tau_{w}}{\tau_{w}}+\frac{\Delta C}{C}+\frac{\Delta u}{u}+\frac{\Delta h}{h}$. Each term of this uncertainty analysis will be considered separately in the following:

- Because of the complex geometry of the flow cell, the correlation given by eq. (6) only provides a crude estimate of the wall shear stress. We performed a simple CFD analysis of 
the flow cell and Fig. 6 (right-hand side) shows the corresponding shear stress distribution on the lower wall. On average, the shear stress is $0.02 \mathrm{~Pa}$ on the lower wall with a peak at approximately $0.037 \mathrm{~Pa}$. Accordingly, depending on where precisely the droplets were located in the flow cell, they could experience a shear stress which is two to three times greater than that predicted by eq. (6). Consequently, a conservative estimate gives $\frac{\Delta \tau_{w}}{\tau_{w}} \sim 1$.

- The constant $C$ which relates the wall shear stress to the effective, averaged shear stress experienced by the droplet is known to vary between 0.118 for a small contact angle of $19^{\circ}$ to 10.2 for a large contact angle $\pi$ (a sphere in shear flow), see (Pozrikidis 1997). The contact angle was shown to vary between 58 and 95 degrees in (Renbaum-Wolff, Grayson, and Bertram 2013). The corresponding uncertainty on $C$ using the data of (Pozrikidis 1997) is $\frac{\Delta C}{C}=0.3$.

- The measured bead velocity in experiments is dependent on where the bead is located within the droplet with beads closer to the free surface travelling faster. CFD simulations were performed and the trajectories of tracer particles within the droplet was followed from which statistics could be inferred. The statistics revealed that the standard deviation on the measured particle velocity was around $25 \%$ of the mean, i.e. $\frac{\Delta u}{u} \sim 25 \%$.

- Finally, the particle sizes was $20 \mu \mathrm{m}$ with a possible deviation of around $5 \mu \mathrm{m}$. This again leads to $\frac{\Delta h}{h} \sim 25 \%$.

This simple uncertainty analysis shows that the $47.9 \%$ error for the highest viscosity can easily be accounted for through the uncertainty of the various quantities involved.

Beside experimental uncertainty, the simple model presented in Section 2 also has limitations since it is based on the lubrication approximation which holds for creeping flow conditions and interfaces with a small slope. Moreover, the analysis is based on a plane flow assumption which does not truly represent the spherical cap geometry assumed in (Pozrikidis 1997) to estimate the constant $C$ of eqs. (7)-(9). The above parametric study provides an estimate of the analytical model imperfection.

In spite of the aforementioned measurement uncertainties and limitations of the model, it should be stressed that the error in the viscosity estimation is smaller than many other methods to assess the viscosity of organic aerosol particles recently reviewed in (Reid et al. 2018).

\section{Conclusions}

A simple model to describe the inner flow in a droplet exposed to an outer shear is described here. The validity of this simple model was first tested against the results from a numerical simulation performed in the commercial Finite Element package COMSOL. A parametric study was performed and results were found to be in reasonable agreement. Importantly, the analytical correlation between the droplet apex velocity and viscosity correlate well with the numerical results confirming the prospect that the velocity can be used as an indirect proxy to evaluate the droplet viscosity. The simple model was then used to assess its ability to replicate data from Refs. (Renbaum-Wolff, Grayson, and Bertram 2013, Renbaum-Wolff et al. 2013). The model was found to predict reasonably well the experimental data especially for lower values of the viscosity with difference between predicted and measured velocity varying between $4.7 \%$ to $47.9 \%$. The difference between the model and the experiment can be attributed to several reasons: uncertainty in the droplet size, droplet contact angle, wall shear stress, unquantified correlation between average bead velocity and apex velocity, and model over-simplification. In spite of these differences, the model offers important insight into the flow physics and importantly potentially circumvent the need to generate 
a calibration curve. It is also anticipated that these results will inform the future design of flow cells and the corresponding testing protocol. Based on the current analysis, a parallel plate flow cell producing a constant and predictable wall shear stress would be much preferable. Using the maximum bead velocity as a proxy is also more likely to match an analytical correlation based on the apex velocity.

\section{Acknowledgements}

The financial support of the College of Engineering at the University of Canterbury through the Strategic Research Grant is gratefully acknowledged. The authors would also like to thank the great efforts of the editor and the referees to improve the quality of our manuscript.

\section{Appendix}

The table below show the results of the parametric study. All parameters are identical to the baseline study of Section 3.a but the $R e$ is altered by changing the inlet gas velocity, the viscosity ratio $\lambda$ by changing the droplet viscosity, and the contact angle by changing the circular segment in order to keep the volume $A$ constant but modify the contact angle.

\begin{tabular}{|c|c|c|c|c|c|}
\hline$R e$ & $\theta$ & $\lambda=\frac{\mu}{\mu_{g}}$ & $\begin{array}{l}u_{\text {apex }}^{\text {num }}(\mathrm{m} / \mathrm{s}) \\
\text { (numerical) }\end{array}$ & $\begin{array}{l}\text { uapex }_{\text {ana }}(\mathrm{m} / \mathrm{s}) \\
\text { (analytical) }\end{array}$ & $\frac{u_{\text {apex }}^{\text {ana }}}{u_{\text {apex }}^{\text {num }}}$ \\
\hline $6.62 \times 10^{-2}$ & $\pi / 2$ & $4.36 \times 10^{4}$ & $3.51 \times 10^{-7}$ & $5.00 \times 10^{-7}$ & 1.42 \\
\hline $3.31 \times 10^{-1}$ & $\pi / 2$ & $4.36 \times 10^{4}$ & $1.76 \times 10^{-6}$ & $2.50 \times 10^{-6}$ & 1.42 \\
\hline $6.62 \times 10^{-1}$ & $\pi / 2$ & $4.36 \times 10^{4}$ & $3.51 \times 10^{-6}$ & $5.00 \times 10^{-6}$ & 1.42 \\
\hline 3.31 & $\pi / 2$ & $4.36 \times 10^{4}$ & $1.77 \times 10^{-5}$ & $2.50 \times 10^{-5}$ & 1.41 \\
\hline 6.62 & $\pi / 2$ & $4.36 \times 10^{4}$ & $3.61 \times 10^{-5}$ & $5.00 \times 10^{-5}$ & 1.39 \\
\hline $5.30 \times 10^{-2}$ & $\pi / 3$ & $4.36 \times 10^{4}$ & $2.50 \times 10^{-7}$ & $2.74 \times 10^{-7}$ & 1.10 \\
\hline $2.65 \times 10^{-1}$ & $\pi / 3$ & $4.36 \times 10^{4}$ & $1.25 \times 10^{-6}$ & $1.37 \times 10^{-6}$ & 1.10 \\
\hline $5.30 \times 10^{-1}$ & $\pi / 3$ & $4.36 \times 10^{4}$ & $2.50 \times 10^{-6}$ & $2.74 \times 10^{-6}$ & 1.10 \\
\hline 2.65 & $\pi / 3$ & $4.36 \times 10^{4}$ & $1.26 \times 10^{-5}$ & $1.37 \times 10^{-5}$ & 1.09 \\
\hline 5.30 & $\pi / 3$ & $4.36 \times 10^{4}$ & $2.54 \times 10^{-5}$ & $2.74 \times 10^{-5}$ & 1.08 \\
\hline $3.69 \times 10^{-2}$ & $\pi / 6$ & $4.36 \times 10^{4}$ & $1.78 \times 10^{-7}$ & $1.33 \times 10^{-7}$ & 0.75 \\
\hline $1.85 \times 10^{-1}$ & $\pi / 6$ & $4.36 \times 10^{4}$ & $8.90 \times 10^{-7}$ & $6.64 \times 10^{-7}$ & 0.75 \\
\hline $3.69 \times 10^{-1}$ & $\pi / 6$ & $4.36 \times 10^{4}$ & $1.78 \times 10^{-6}$ & $1.33 \times 10^{-6}$ & 0.75 \\
\hline 1.85 & $\pi / 6$ & $4.36 \times 10^{4}$ & $8.91 \times 10^{-6}$ & $6.64 \times 10^{-6}$ & 0.74 \\
\hline 3.69 & $\pi / 6$ & $4.36 \times 10^{4}$ & $1.79 \times 10^{-5}$ & $1.33 \times 10^{-5}$ & 0.75 \\
\hline $6.62 \times 10^{-2}$ & $\pi / 2$ & $4.36 \times 10^{3}$ & $3.52 \times 10^{-6}$ & $5.00 \times 10^{-6}$ & 1.42 \\
\hline $3.31 \times 10^{-1}$ & $\pi / 2$ & $4.36 \times 10^{3}$ & $1.76 \times 10^{-5}$ & $2.50 \times 10^{-5}$ & 1.42 \\
\hline $6.62 \times 10^{-1}$ & $\pi / 2$ & $4.36 \times 10^{3}$ & $3.52 \times 10^{-5}$ & $5.00 \times 10^{-5}$ & 1.42 \\
\hline 3.31 & $\pi / 2$ & $4.36 \times 10^{3}$ & $1.78 \times 10^{-4}$ & $2.50 \times 10^{-4}$ & 1.41 \\
\hline 6.62 & $\pi / 2$ & $4.36 \times 10^{3}$ & $3.64 \times 10^{-4}$ & $5.00 \times 10^{-4}$ & 1.38 \\
\hline $5.30 \times 10^{-2}$ & $\pi / 3$ & $4.36 \times 10^{3}$ & $2.50 \times 10^{-6}$ & $2.74 \times 10^{-6}$ & 1.10 \\
\hline $2.65 \times 10^{-1}$ & $\pi / 3$ & $4.36 \times 10^{3}$ & $1.25 \times 10^{-5}$ & $1.37 \times 10^{-5}$ & 1.10 \\
\hline $5.30 \times 10^{-1}$ & $\pi / 3$ & $4.36 \times 10^{3}$ & $2.50 \times 10^{-5}$ & $2.74 \times 10^{-5}$ & 1.10 \\
\hline 2.65 & $\pi / 3$ & $4.36 \times 10^{3}$ & $1.26 \times 10^{-4}$ & $1.37 \times 10^{-4}$ & 1.09 \\
\hline 5.30 & $\pi / 3$ & $4.36 \times 10^{3}$ & $2.54 \times 10^{-4}$ & $2.74 \times 10^{-4}$ & 1.08 \\
\hline
\end{tabular}




\begin{tabular}{|r|c|r|r|r|r|}
\hline $3.69 \times 10^{-2}$ & $\pi / 6$ & $4.36 \times 10^{3}$ & $1.78 \times 10^{-6}$ & $1.33 \times 10^{-6}$ & 0.75 \\
\hline $1.85 \times 10^{-1}$ & $\pi / 6$ & $4.36 \times 10^{3}$ & $8.90 \times 10^{-6}$ & $6.64 \times 10^{-6}$ & 0.75 \\
\hline $3.69 \times 10^{-1}$ & $\pi / 6$ & $4.36 \times 10^{3}$ & $1.78 \times 10^{-5}$ & $1.33 \times 10^{-5}$ & 0.75 \\
\hline 1.85 & $\pi / 6$ & $4.36 \times 10^{3}$ & $8.91 \times 10^{-5}$ & $6.64 \times 10^{-5}$ & 0.75 \\
\hline 3.69 & $\pi / 6$ & $4.36 \times 10^{3}$ & $1.79 \times 10^{-4}$ & $1.33 \times 10^{-4}$ & 0.74 \\
\hline $6.62 \times 10^{-2}$ & $\pi / 2$ & $4.36 \times 10^{5}$ & $3.51 \times 10^{-8}$ & $5.00 \times 10^{-8}$ & 1.42 \\
\hline $3.31 \times 10^{-1}$ & $\pi / 2$ & $4.36 \times 10^{5}$ & $1.76 \times 10^{-7}$ & $2.50 \times 10^{-7}$ & 1.42 \\
\hline $6.63 \times 10^{-1}$ & $\pi / 2$ & $4.36 \times 10^{5}$ & $3.51 \times 10^{-7}$ & $5.00 \times 10^{-7}$ & 1.42 \\
\hline 3.31 & $\pi / 2$ & $4.36 \times 10^{5}$ & $1.77 \times 10^{-6}$ & $2.50 \times 10^{-6}$ & 1.41 \\
\hline 6.62 & $\pi / 2$ & $4.36 \times 10^{5}$ & $3.60 \times 10^{-6}$ & $5.00 \times 10^{-6}$ & 1.39 \\
\hline $5.30 \times 10^{-2}$ & $\pi / 3$ & $4.36 \times 10^{5}$ & $2.50 \times 10^{-8}$ & $2.74 \times 10^{-8}$ & 1.10 \\
\hline $2.65 \times 10^{-1}$ & $\pi / 3$ & $4.36 \times 10^{5}$ & $1.25 \times 10^{-7}$ & $1.37 \times 10^{-7}$ & 1.10 \\
\hline $5.30 \times 10^{-1}$ & $\pi / 3$ & $4.36 \times 10^{5}$ & $2.50 \times 10^{-7}$ & $2.74 \times 10^{-7}$ & 1.10 \\
\hline 2.65 & $\pi / 3$ & $4.36 \times 10^{5}$ & $1.26 \times 10^{-6}$ & $1.37 \times 10^{-6}$ & 1.09 \\
\hline 5.30 & $\pi / 3$ & $4.36 \times 10^{5}$ & $2.54 \times 10^{-6}$ & $2.74 \times 10^{-6}$ & 1.08 \\
\hline $3.69 \times 10^{-2}$ & $\pi / 6$ & $4.36 \times 10^{5}$ & $1.78 \times 10^{-8}$ & $1.33 \times 10^{-8}$ & 0.75 \\
\hline $1.85 \times 10^{-1}$ & $\pi / 6$ & $4.36 \times 10^{5}$ & $8.90 \times 10^{-8}$ & $6.64 \times 10^{-8}$ & 0.75 \\
\hline $3.69 \times 10^{-1}$ & $\pi / 6$ & $4.36 \times 10^{5}$ & $1.78 \times 10^{-7}$ & $1.33 \times 10^{-7}$ & 0.75 \\
\hline 1.85 & $\pi / 6$ & $4.36 \times 10^{5}$ & $8.81 \times 10^{-7}$ & $6.64 \times 10^{-7}$ & 0.75 \\
\hline 3.69 & $\pi / 6$ & $4.36 \times 10^{5}$ & $1.79 \times 10^{-6}$ & $1.33 \times 10^{-6}$ & 0.74 \\
\hline
\end{tabular}

Table 2: Parametric study results comparing the apex droplet velocity computed numerically and that from the analytical model

\section{References}

Dussan, EB. 1987. "On the ability of drops to stick to surfaces of solids. 3 . The influences of the motion of the surrounding fluid on dislodging drops." J. Fluid Mech. 174:381-397.

Fan, J, MCT Wilson, and N Kapur. 2011. "Displacement of liquid droplets on a surface by a shearing air flow." Journal of colloid and interface science 356 (1):286-292.

Grayson, JW, M Song, M Sellier, and AK Bertram. 2015. "Validation of the poke-flow technique combined with simulations of fluid flow for determining viscosities in samples with small volumes and high viscosities." Atmospheric Measurement Techniques 8 (6):2463.

Han, Zuoyan, Xiaoju Tang, and Bo Zheng. 2007. "A PDMS viscometer for microliter Newtonian fluid." Journal of Micromechanics and Microengineering 17 (9):1828.

Hosny, Neveen A, Clare Fitzgerald, Changlun Tong, Markus Kalberer, Marina K Kuimova, and Francis D Pope. 2013. "Fluorescent lifetime imaging of atmospheric aerosols: a direct probe of aerosol viscosity." Faraday discussions 165:343-356.

Huh, Chun, and LE Scriven. 1971. "Hydrodynamic model of steady movement of a solid/liquid/fluid contact line." Journal of Colloid and Interface Science 35 (1):85-101.

Järvinen, Emma, Karoliina Ignatius, Leonid Nichman, Thomas B Kristensen, Claudia Fuchs, Christopher R Hoyle, Niko Höppel, Joel C Corbin, Jill Craven, and Jonathan Duplissy. 2016. "Observation of viscosity transition in $\alpha$-pinene secondary organic aerosol." Atmospheric Chemistry and Physics 16 (7):4423-4438.

LeClair, BP, AE Hamielec, HR Pruppacher, and WD Hall. 1972. "A theoretical and experimental study of the internal circulation in water drops falling at terminal velocity in air." Journal of the Atmospheric Sciences 29 (4):728-740. 
Mahé, M, M Vignes-Adler, A Rousseau, CG Jacquin, and PM Adler. 1988. "Adhesion of droplets on a solid wall and detachment by a shear flow: I. Pure systems." Journal of colloid and interface science 126 (1):314-328.

Müller, Francisco J, and Julio C Pita. 1983. "A simple electronic capillary microviscometer." Analytical biochemistry 135 (1):106-111.

Perraud, Véronique, Emily A Bruns, Michael J Ezell, Stanley N Johnson, Yong Yu, M Lizabeth Alexander, Alla Zelenyuk, Dan Imre, Wayne L Chang, and Donald Dabdub. 2012. "Nonequilibrium atmospheric secondary organic aerosol formation and growth." Proceedings of the National Academy of Sciences 109 (8):2836-2841.

Pipe, Christopher J, and Gareth H McKinley. 2009. "Microfluidic rheometry." Mechanics Research Communications 36 (1):110-120.

Power, Rory M, and Jonathan P Reid. 2014. "Probing the micro-rheological properties of aerosol particles using optical tweezers." Reports on Progress in Physics 77 (7):074601.

Pozrikidis, C. 1997. "Shear flow over a protuberance on a plane wall." Journal of Engineering Mathematics 31 (1):29-42.

Reid, Jonathan P, Allan K Bertram, David O Topping, Alexander Laskin, Scot T Martin, Markus D Petters, Francis D Pope, and Grazia Rovelli. 2018. "The viscosity of atmospherically relevant organic particles." Nature communications 9 (1):956.

Renbaum-Wolff, L, JW Grayson, and AK Bertram. 2013. "New methodology for measuring viscosities in small volumes characteristic of environmental chamber particle samples." Atmospheric Chemistry and Physics 13 (2):791-802.

Renbaum-Wolff, Lindsay, James W Grayson, Adam P Bateman, Mikinori Kuwata, Mathieu Sellier, Benjamin J Murray, John E Shilling, Scot T Martin, and Allan K Bertram. 2013. "Viscosity of $\alpha$ pinene secondary organic material and implications for particle growth and reactivity." Proceedings of the National Academy of Sciences 110 (20):8014-8019.

Rothfuss, Nicholas E, and Markus D Petters. 2016. "Coalescence-based assessment of aerosol phase state using dimers prepared through a dual-differential mobility analyzer technique." Aerosol Science and Technology 50 (12):1294-1305.

Shiraiwa, Manabu, Markus Ammann, Thomas Koop, and Ulrich Pöschl. 2011. "Gas uptake and chemical aging of semisolid organic aerosol particles." Proceedings of the National Academy of Sciences 108 (27):11003-11008.

Solomon, Susan, D Qin, M Manning, Z Chen, M Marquis, KB Averyt, MMB Tignor, and HL Miller. 2007. Climate change 2007: the physical science basis. Contribution of Working Group I to the Fourth Assessment Report of the Intergovernmental Panel on Climate Change, 2007.

Song, M, PF Liu, SJ Hanna, YJ Li, ST Martin, and AK Bertram. 2015. "Relative humidity-dependent viscosities of isoprene-derived secondary organic material and atmospheric implications for isoprene-dominant forests." Atmospheric Chemistry and Physics 15 (9):5145-5159.

Song, Mijung, Pengfei F Liu, Sarah J Hanna, Rahul A Zaveri, Katie Potter, Yuan You, Scot T Martin, and Allan K Bertram. 2016. "Relative humidity-dependent viscosity of secondary organic material from toluene photo-oxidation and possible implications for organic particulate matter over megacities." Atmospheric Chemistry and Physics 16 (14):8817-8830.

Spelt, Peter DM. 2006. "Shear flow past two-dimensional droplets pinned or moving on an adhering channel wall at moderate Reynolds numbers: a numerical study." Journal of Fluid Mechanics 561:439-463.

Srivastava, Nimisha, and Mark A Burns. 2006. "Analysis of non-Newtonian liquids using a microfluidic capillary viscometer." Analytical chemistry 78 (5):1690-1696.

Sugiyama, K, and M Sbragaglia. 2008. "Linear shear flow past a hemispherical droplet adhering to a solid surface." Journal of engineering mathematics 62 (1):35-50.

Virtanen, Annele, Jorma Joutsensaari, Thomas Koop, Jonna Kannosto, Pasi Yli-Pirilä, Jani Leskinen, Jyrki M Mäkelä, Jarmo K Holopainen, Ulrich Pöschl, and Markku Kulmala. 2010. "An 
amorphous solid state of biogenic secondary organic aerosol particles." Nature 467 (7317):824.

Wang, Bingbing, Andrew T Lambe, Paola Massoli, Timothy B Onasch, Paul Davidovits, Douglas R Worsnop, and Daniel A Knopf. 2012. "The deposition ice nucleation and immersion freezing potential of amorphous secondary organic aerosol: Pathways for ice and mixed-phase cloud formation." Journal of Geophysical Research: Atmospheres 117 (D16).

Woodhouse, Francis G, and Raymond E Goldstein. 2012. "Shear-driven circulation patterns in lipid membrane vesicles." Journal of Fluid Mechanics 705:165-175.

Zaveri, Rahul A, John E Shilling, Alla Zelenyuk, Jiumeng Liu, David M Bell, Emma L D’Ambro, Cassandra J Gaston, Joel A Thornton, Alexander Laskin, and Peng Lin. 2018. "Growth kinetics and size distribution dynamics of viscous secondary organic aerosol." Environmental science \& technology 52 (3):1191-1199.

Zhang, Y, MS Sanchez, C Douet, Y Wang, AP Bateman, Z Gong, Mikinori Kuwata, L Renbaum-Wolff, BB Sato, and PF Liu. 2015. "Changing shapes and implied viscosities of suspended submicron particles." Atmospheric Chemistry and Physics 15 (14):7819-7829. 